|| ISSN(online): 2589-8698 || ISSN(print): 2589-868X || International Journal of Medical and Biomedical Studies

Available Online at www.ijmbs.info

PubMed (National Library of Medicine ID: 101738825)

Index Copernicus Value 2018: 75.71

Volume 3, Issue 4; April: 2019; Page No. 15-26

\title{
MOTOR IMAGERY TOGETHER WITH CONVENTIONAL THERAPY WILL BE EQUAL TO OR MORE EFFECTIVE THAN CONVENTIONAL ALONE FOR IMPROVEMENT OF GAIT IN STROKE PATIENT
}

\author{
${ }^{1}$ Dr. Barkha Khurana, ${ }^{2}$ Dr. Ashish Dobhal and Dr. Vishesh Singhal \\ ${ }^{1}$ Director, Faculty of Physiotherapy \& Diagnostics, Jayoti Vidyapeeth Women University, Jaipur \\ ${ }^{2}$ Assistant Professor, Faculty of Physiotherapy \& Diagnostics, Jayoti Vidyapeeth Women University, \\ Jaipur. \\ ${ }^{3}$ Assistant Professor, Physiotherapy Department Chandigarh University, Punjab
}

Article Info: Received 18 February 2019; Accepted 02 April. 2019

Cite this article as: Khurana, Dr. B., Dobhal, Dr. A., \& Singhal, Dr. V. (2019). MOTOR IMAGERY TOGETHER WITH

CONVENTIONAL THERAPY WILL BE EQUAL TO OR MORE EFFECTIVE THAN CONVENTIONAL ALONE FOR

IMPROVEMENT OF GAIT IN STROKE PATIENT. International Journal of Medical and Biomedical Studies, 3(4).

DOI: https://doi.org/10.32553/ijmbs.v3i4.182

Address for Correspondence: Dr. Barkha Khurana, Director, Faculty of Physiotherapy \& Diagnostics, Jayoti Vidyapeeth Women University, Jaipur

Conflict of interest: No conflict of interest.

\section{Abstract}

Strokes can result in many different disabilities, ranging from motor control and urinary incontinence to depression and memory loss. Stroke usually occurs on only one side of the brain, so decreased motor control (the ability to move muscles in a coordinated manner) usually develops on only one side of the body. Total 30 participants including both male and female who were previously diagnosed by Neurologist was recruited for the study. Subject will be selected as per convenient sampling and assigned into two groups i.e. Group -A (Experimental group) and Group-B (Control Group). In group-A subjects were given motor imagery and conventional therapy both; in group-B subjects were given conventional therapy alone. This group received Motor imagery (10-15 minute) \& Conventional therapy (30-40 minutes) both and it was given in single session of 45-50 minutes. The program was conducted for 3 times per week. Total duration of both programs was for 4 weeks. For group B Intervention of Conventional therapy alone was given for 30-40 min. No significant differences were found between the groups regarding their age. Group A got 13 subjects with (mean age $=65.46$ years \pm 7.55 ) and Group B got 13 subjects with (mean age $=65.69 \pm 5.58$ ). Both programs were conducted in the respective participant's home and hospital environment. The procedure requires only a stopwatch, two felt tip marking pens with washable ink, and a $16-\mathrm{m}(53 \mathrm{ft})$ walkway that is premeasured and marked with masking tape at four points. Initially subjects were introduced with Motor Imagery technique. Statistics are performed by using SPSS 13 and Sigma state. t- Test was used for analysis of data. Paired t-test was applied to compare the data of Gait assessment (stride length, step length, gait velocity and cadence) within group. Student t-test was used to compare the data of gait assessment (stride length, step length, gait velocity and cadence) between groups. The statistical significance was set at 0.05 at $95 \%$ confidence interval and $P$ value $<0.05$ was considered significant.

Key words: Strokes, Gait assessment, Motor Imagery and Conventional therapy. 


\section{INTRODUCTION:}

Strokes can result in many different disabilities, ranging from motor control and urinary incontinence to depression and memory loss. Stroke usually occurs on only one side of the brain, so decreased motor control (the ability to move muscles in a coordinated manner) usually develops on only one side of the body. In fact, one side of the body may be paralyzed (hemiplegia), or muscles on the affected side may be weakened (hemiparesis). Because of the weakness or paralysis in large muscle groups, injuries from falls are common complications of motor control disturbances ${ }^{2}$

Functional magnetic resonance imaging (fMRI) showed activation in frontal, parietal cortical and sub-cortical areas that are involved in action planning, execution and modulation. Recently, the first fMRI study was published that investigated brain activation during imagination of whole body movements, supporting the findings from many $\mathrm{Ml}$ intervention studies in sport psychology 6 . Functional brain imaging studies have indicated that several cortical and subcortical areas active during actual motor performance are also active during imagination or mental rehearsal of movements ${ }^{9}$

In the absence of the movement, there is detectable EMG activity during motor imagery, this shows there is a cortical excitability with no changes in spinal excitability ${ }^{7}$. Motor imagery is a high level process which however manifests itself in the activation of those same cortical circuits that are normally involved in the movement execution ${ }^{8}$. Imagery practice should focus on its specific impairments during gait in order to affect the performance of the paretic lower extremity with conventional therapy ${ }^{4-12}$.

\section{METHODOLOGY}

\subsection{Sample}

Total 30 participants including both male and female who were previously diagnosed by Neurologist was recruited for the study.

\subsection{Source of Subject}

Subjects were taken from:

Institute of Liver and Biliary Sciences Hospital, New Delhi

Fortis Escorts Heart Institute \& Research Centre Hospital, Okhla Road, New Delhi

Saket City Hospital, Saket, New Delhi

Bhopal Memorial Hospital \& Research Centre (BMHRC), Bhopal (M.P.)

Narmada Trauma Centre, Bhopal (M.P.)

\subsection{Study Design}

\subsubsection{Method of Selecting and Assigning Subject}

Subject will be selected as per convenient sampling and assigned into two groups i.e. Group -A (Experimental group) and Group-B (Control Group). In group-A subjects were given motor imagery and conventional therapy both; in group-B subjects were given conventional therapy alone.

\subsubsection{Inclusion Criteria}

1. Ambulatory Stroke patients. Can ambulate 16 meter (with or without assistive device.

2. Stroke of at least 3 months duration.

3. No serious unstable medical complication.

4. Not receiving any other form of physiotherapy for lower limb.

5. Mini-mental state examination. (> 23).

6. Movement Imagery Questionnaire - Revised second (MIQ-RS): (score of 98 is good, score of 14 is worst.)

\subsubsection{Exclusion Criteria}

1. Spinal deformity.

2. History of spinal trauma or head injury.

3. Any other neurological disease.

4. Unhealed Fractures.

5. Peripheral arterial occlusive disease.

6. Orthopaedic disorder involving any joint of lower limbs.

7. History of neurologic disease other than the chronic stroke

\subsection{Instrumentation}

1. Plinth or couch. (Performing motor imagery) 
2. Stopwatch. (For evaluation of gait velocity)

3. Plain surface for walk test (at least $16 \mathrm{~m}$ )

4. Chart paper. $(6+6 \mathrm{~m}$ per subject)

5. White board marker (for heel strike mark)

6. Adhesive tape \& double tape (for attachment of marker with shoe)

7. Inch/Measuring tape (measurement of space and chart)

8. Scale (measurement of step length and stride length)

\subsection{Outcome Measure}

1. Stride length measurement: (the average of middle three strides) $\mathrm{cm}$

2. Step length measurement: (the average of the middle three steps) $\mathrm{cm}$

3. Gait velocity measurement: $(6 \mathrm{~m} \times 60 \mathrm{sec} \div$ time for walk in sec) $\mathrm{m} / \mathrm{min}$

4. Cadence measurement: (\# marks $\times 60 \div$ time for walk in sec) steps/min

\subsection{Protocol}

This study consisted of two groups- Experimental group (A) and Control group (B). 26 subjects were chosen as per the inclusion and exclusion criteria, and informed consent was obtained from all subjects after the procedure was explained to them.

The 4 weeks intervention was given to the subjects of both groups alternately, 3days a week for Group A and 3days a week for group B. 45-50 minutes protocol for Group A and 30-40 minutes protocol for Group B.

\subsubsection{Group-A Protocol}

This group received Motor imagery (10-15 minute) \& Conventional therapy (30-40 minutes) both and it was given in single session of 45-50 minutes. The program was conducted for 3 times per week. Total duration of both programs was for 4 weeks.

Motor Imagery technique used: The internal as well as external imagery scenes were applied in this intervention protocol. The 2 main goals were:

- To facilitate movement and posture of the affected lower extremity during gait by focusing on specific impairments

- To enhance functional walking within subjects own environment

Conventional therapy technique used: The Conventional therapy for gait training in this group was given as per the protocol of Group B.

\subsubsection{Group-B Protocol}

In this group Intervention of Conventional therapy alone was given for 30-40 min. In Conventional gait training, Patient practiced functional,

Task-specific loco motor skill walking forward and side stepping) (5 minutes)

Elevation activities (e.g. step-up/step-down, lateral step-up, stair climbing,)

Community activities (walking on ramps, curves and over and around obstacles), and Quadriceps strengthening. ${ }^{48}$

\subsection{Procedure}

A total of 26 subjects who were previously diagnosed as cases of Stroke by a Neurologist and met the inclusion criteria of the study were included in the study. The participants were screened by - mini mental state examination, before they were selected for the study. Prior to enrolling into the study, need and purpose of study were told to the participants. Informed consent was signed before study. Having given informed consent, subjects were allocated by convenient sampling into Motor Imagery \& Conventional therapy both i.e. GROUP $A$ and Conventional therapy alone i.e. GROUP B. Participants who were unable to perform or understand Motor Imagery task were excluded from Groups. They were allowed to terminate their participation at any time during study. There were 2 groups each with 26 participants. After obtaining a written consent form, demographic data were collected. No significant differences were found between the groups regarding their age. Group A got 13 subjects with (mean age $=65.46$ years \pm 7.55 ) and Group B got 
13 subjects with (mean age $=65.69 \pm 5.58$ ). Both programs were conducted in the respective participant's home and hospital environment. None of the subjects attended physiotherapy for lower limb anywhere else during the study. Baseline measurement was taken at the start of treatment program, using gait variables as outcome measures i.e. stride length, Step length, Gait velocity \& Cadence.

\subsection{Gait Assessment}

The procedure requires only a stopwatch, two felt tip marking pens with washable ink, and a $16-\mathrm{m}$ (53ft) walkway that is premeasured and marked with masking tape at four points. A hallway, an outside cement area at a clinic, or patient's home, as well as a portion of a clinic floor can be used for the walkway. The walkway is marked to show a centre area $6 \mathrm{~m}$ long and two 5-m areas on each end. Measurements are made within the 6-m area only; the two 5-m areas allow for warming up to "normal" velocity before measurement and slowing down after measurement. Using these extensions of the measurement area of the walkway is intended to eliminate measurement errors.

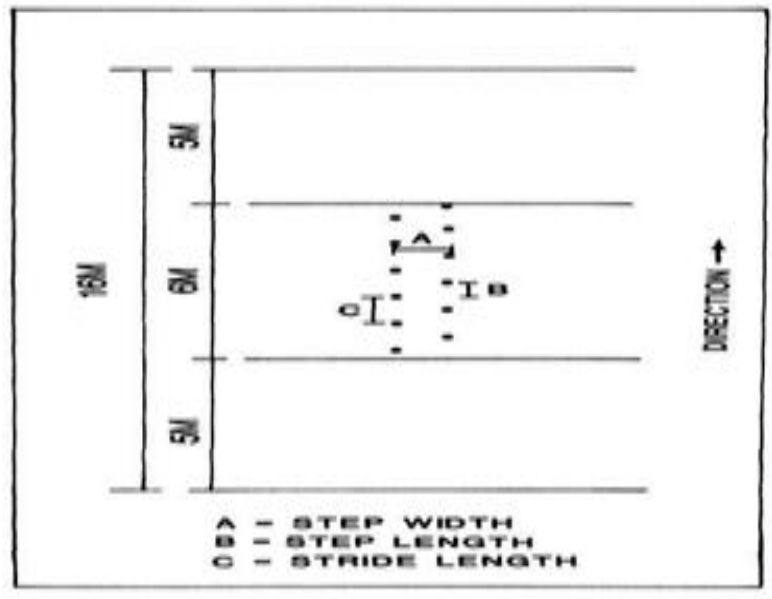

Figure 1: Shows pattern of steps

The patient is instructed to walk at his usual walking speed from one end of the $16-\mathrm{m}$ walkway to the other end. The therapist, using a stopwatch, records the time taken for the patient to walk the centre $6 \mathrm{~m}$. Measurements within the 6-m area are then made of distances from each heel contact pen mark to the next heel contact pen mark on the same side (stride length) and step length was taken with stance of affected side. (Sometimes the marker leaves a line mark as the heel nears the floor for contact. The point at the termination of the line mark should be used for measurement.) Also, the total number of contact marks in the centre $6 \mathrm{~m}$ is counted.

\subsection{Motor Imagery Technique}

For training gait by motor imagery therapist/myself was standing in front of subject who was sitting on the chair with arm rest or lying on bed as per the comfort of subject. Initially subjects were introduced with Motor Imagery technique. They were well explained about it.

\subsubsection{Conventional Therapy for Gait}

Walking: Subject standing with hip in correct alignment, subject practices stepping forward then backward with intact leg, making sure he/she extends his/her affected hip as he/she steps forward. I stand on either in front or on affected side and encourage the subject to take weight through affected leg. Likewise instruct to subject for forward walking. Subject was instructed for side walking by hip abduction and takes a long step at one side and follow by other step.

Elevation Activities: Subject was instructed to take a step-up on stairs by flexing hip and knee, with giving load on the forward limb and elevate his/her body to climb the one stair up. Then subject was instructed for step-down the stair by hip extension of one limb and hip flexion and knee flexion of other. Assistance was required where subject is not able to perform the activity due to fear of falling. Likewise subject was instructed stair climbing.

Community Activities: Subject performs walking on ramps, curves and uneven terrain as this increases the gait speed by increasing endurance. The over and around obstacles task was also given to the subject by placing a stick in 
front of subject and instructed for walk over the stick.

Quadriceps Strengthening: Resistive strength training was given to subject by tying weight cuff to the foot, and performing extension of knee.

\section{RESULT AND DISCUSSION:}

Statistics are performed by using SPSS 13 and Sigma state. $t$ - Test was used for analysis of data. Paired t-test was applied to compare the data of Gait assessment (stride length, step length, gait velocity and cadence) within group. Student ttest was used to compare the data of gait assessment (stride length, step length, gait velocity and cadence) between groups. The statistical significance was set at 0.05 at $95 \%$ confidence interval and $P$ value $<0.05$ was considered significant.

Student T-test was done to compare the data of pre stride length between the groups. $(p=0.928)$ [Table-1]

Table 1: Shows Mean and SD of Pre-Stride length (SDL) for Group-A and Group-B

\begin{tabular}{|l|c|c|c|c|}
\hline SDL & \multicolumn{2}{|c|}{ Group-A } & Group-B \\
\hline \multirow{3}{*}{ PRE } & Mean & SD & Mean & SD \\
\cline { 2 - 5 } & 65.03 & 15.08 & 64.46 & 17.14 \\
\cline { 2 - 5 } & $\mathrm{t}=.091$ & \multicolumn{4}{|l}{} \\
\cline { 2 - 5 } & $\mathrm{P}=.928$ & \\
\end{tabular}

Paired t-test was done to compare the data of Stride length within the groups. For Stride length both the groups showed significant difference [Group A ( $p=0.023)$ and Group B ( $p=0.000)$ [Table-2].

Table 2: Shows Mean and SD of Pre-Stride length (SDL) \& Post-Stride length (SDL) for Group-A and Group-B

\begin{tabular}{|c|c|c|c|c|c|c|c|}
\hline \multicolumn{4}{|c|}{ Group-A } & \multicolumn{4}{|c|}{ Group-B } \\
\hline \multicolumn{4}{|l|}{ SDL } & \multicolumn{4}{|l|}{ SDL } \\
\hline \multicolumn{2}{|l|}{ PRE } & \multicolumn{2}{|l|}{ POST } & \multicolumn{2}{|l|}{ PRE } & \multicolumn{2}{|l|}{ POST } \\
\hline Mean & SD & Mean & SD & Mean & SD & Mean & SD \\
\hline 65.03 & 15.08 & 79.00 & 24.41 & 64.46 & 17.14 & 74.301 & 19.3 \\
\hline \multicolumn{4}{|c|}{$t=2.629$} & \multicolumn{4}{|c|}{$t=5.009$} \\
\hline \multicolumn{4}{|c|}{$P=0.023$} & \multicolumn{4}{|c|}{$P=0.000$} \\
\hline
\end{tabular}

Student T-test was done to compare the data of post stride length between the groups. $(p=0.592)$ [Table-3]

Table 3: Shows Mean and SD of Post-Stride length (SDL) for Group-A and Group-B

\begin{tabular}{|c|c|c|c|c|}
\hline SDL & \multicolumn{2}{|c|}{ Group-A } & Group-B \\
\hline \multirow{3}{*}{ POST } & Mean & SD & Mean & SD \\
\cline { 2 - 5 } & 79.00 & 24.4123 & 74.3077 & 19.3052 \\
\cline { 2 - 5 } & $\mathrm{t}=.544$ & \multicolumn{4}{|c}{} \\
\cline { 2 - 5 } & $\mathrm{P}=.592$ & \multicolumn{4}{|c}{} \\
\cline { 2 - 5 } &
\end{tabular}

Student T-test was done to compare the data of pre step length between the groups. ( $p=0.777)$ [Table4] 
Table 4: Shows Mean and SD of Pre-Step length (STL) for Group-A and Group-B

\begin{tabular}{|c|c|c|c|c|}
\hline STL & \multicolumn{2}{|c|}{ Group-A } & Group-B \\
\hline \multirow{3}{*}{ PRE } & Mean & SD & Mean & SD \\
\cline { 2 - 5 } & 31.4200 & 7.76 & 31.13 & 7.15 \\
\cline { 2 - 5 } & $\mathrm{t}=-.286$ & \multicolumn{4}{|l}{} \\
\cline { 2 - 5 } & $\mathrm{P}=.777$ & & \\
\end{tabular}

Paired t-test was done to compare the data of Step length within the groups. For Step length both the groups showed significant difference [Group A ( $p=0.024)$ and Group B ( $p=0.002)$ ] [Table-5].

Table 5: Shows Mean and SD of Pre-Step length \& Post-Step length (STL) for Group-A and Group-B

\begin{tabular}{|c|c|c|c|c|c|c|c|}
\hline \multicolumn{4}{|l|}{ Group-A } & \multicolumn{4}{|l|}{ Group-B } \\
\hline \multicolumn{4}{|l|}{ STL } & \multicolumn{4}{|l|}{ STL } \\
\hline \multicolumn{2}{|l|}{ PRE } & \multicolumn{2}{|l|}{ POST } & \multicolumn{2}{|l|}{ PRE } & \multicolumn{2}{|l|}{ POST } \\
\hline Mean & SD & Mean & SD & Mean & SD & Mean & SD \\
\hline 31.42 & 7.7 & 38.17 & 12.09 & 31.13 & 7.15 & 36.07 & 9.2 \\
\hline \multicolumn{4}{|l|}{$t=2.59$} & \multicolumn{4}{|l|}{$t=3.825$} \\
\hline \multicolumn{4}{|l|}{$P=0.024$} & \multicolumn{4}{|l|}{$P=0.002$} \\
\hline
\end{tabular}

Student T-test was done to compare the data of post step length between the groups. $(p=0.631)$ [Table-6]

Table 6: Shows Mean and SD of Post-Step length (STL) for Group-A and Group-B

\begin{tabular}{|c|c|c|c|c|}
\hline STL & \multicolumn{2}{|c|}{ Group-A } & Group-B \\
\hline \multirow{3}{*}{ POST } & Mean & SD & Mean & SD \\
\cline { 2 - 5 } & 38.1754 & 12.5092 & 36.0738 & 9.2997 \\
\cline { 2 - 5 } & $\mathrm{t}=.486$ & \multicolumn{3}{|c}{} \\
\cline { 2 - 5 } & $\mathrm{P}=.631$ & & \\
&
\end{tabular}

Student T-test was done to compare the data of pre Gait velocity between the groups. $(p=0.459)$ [Table-7]

Table 7: Shows Mean and SD of Pre-Gait velocity (VL) for Group-A and Group-B

\begin{tabular}{|c|c|c|c|c|}
\hline VL & \multicolumn{2}{|c|}{ Group-A } & \multicolumn{2}{c|}{ Group-B } \\
\hline \multirow{3}{*}{ PRE } & Mean & SD & Mean & SD \\
\cline { 2 - 5 } & 27.1492 & 10.9446 & 26.824 & 10.0134 \\
\cline { 2 - 5 } & $\mathrm{t}=-.753$ & \multicolumn{3}{|c}{} \\
\cline { 2 - 5 } & $\mathrm{P}=.459$ & \multicolumn{3}{|l}{} \\
\cline { 2 - 5 } &
\end{tabular}

Paired t-test was done to compare the data of Gait velocity within the groups. For Gait velocity both the groups showed significant difference [Group A ( $p=0.015)$ and Group B $(p=0.000)$ ] [Table-8]. 
Table 8: Shows Mean and SD of Pre-Gait velocity \& post-Gait velocity for Group-A and Group-B

\begin{tabular}{|c|c|c|c|c|c|c|c|}
\hline \multicolumn{4}{|l|}{ Group-A } & \multicolumn{4}{|l|}{ Group-B } \\
\hline \multicolumn{4}{|l|}{$\overline{V L}$} & \multicolumn{4}{|l|}{$\mathrm{VL}$} \\
\hline \multicolumn{2}{|l|}{ PRE } & \multicolumn{2}{|l|}{ POST } & \multicolumn{2}{|l|}{ PRE } & \multicolumn{2}{|l|}{ POST } \\
\hline Mean & SD & Mean & SD & Mean & SD & Mean & SD \\
\hline 27.14 & 10.9 & 36.86 & 23.0 & 26.89 & 7.2 & 34.9 & 11.47 \\
\hline \multicolumn{4}{|l|}{$t=2.09$} & \multicolumn{4}{|l|}{$t=4.916$} \\
\hline \multicolumn{4}{|l|}{$P=0.015$} & \multicolumn{4}{|c|}{$P=0.000$} \\
\hline
\end{tabular}

Student T-test was done to compare the data of post Gait velocity between the groups. $(p=0.959)$ [Table-9]

Table 9: Shows Mean and SD of Post-Gait velocity for Group-A and Group-B

\begin{tabular}{|c|c|c|c|c|}
\hline \multirow{2}{*}{ VL } & \multicolumn{2}{|c|}{ Group-A } & \multicolumn{2}{c|}{ Group-B } \\
\hline \multirow{3}{*}{ Post } & Mean & SD & Mean & SD \\
\cline { 2 - 5 } & 36.8615 & 23.0036 & 36.4823 & 13.0434 \\
\cline { 2 - 5 } & $\mathrm{t}=.052$ & \multicolumn{3}{|c}{} \\
\cline { 2 - 5 } & $\mathrm{P}=.959$ & & \\
&
\end{tabular}

Paired t-test was done to compare the data of Cadence within the groups. For Cadence both the groups showed significant difference [Group A ( $p=0.024)$ and Group B ( $p=0.002)$ ] [Table-10].

Table 10: Shows Mean and SD of Pre-Cadence \& Post-Cadence for Group-A and Group-B

\begin{tabular}{|c|c|c|c|c|c|c|c|}
\hline \multicolumn{4}{|c|}{ Group -A } & \multicolumn{4}{|c|}{ Group-B } \\
\hline \multicolumn{4}{|l|}{ CAD } & \multicolumn{4}{|l|}{ CAD } \\
\hline \multicolumn{2}{|l|}{ PRE } & \multicolumn{2}{|l|}{ POST } & \multicolumn{2}{|l|}{ PRE } & \multicolumn{2}{|l|}{ POST } \\
\hline Mean & $S D$ & Mean & SD & Mean & $S D$ & Mean & $S D$ \\
\hline 74.63 & 23.76 & 85.89 & 33.39 & 74.18 & 19.69 & 83.72 & 23.29 \\
\hline \multicolumn{4}{|c|}{$t=2.578$} & \multicolumn{4}{|c|}{$t=3.848$} \\
\hline \multicolumn{4}{|c|}{$P=0.024$} & \multicolumn{4}{|c|}{$P=0.002$} \\
\hline
\end{tabular}

Student T-test was done to compare the data of post cadence between the groups. $(p=0.844)$ [Table11]

Table 11: Shows Mean and SD of Post-Cadence (CAD) for Group-A and Group-B

\begin{tabular}{|c|c|c|c|c|}
\hline CAD & Group-A & & Group-B & \\
\hline \multirow[t]{4}{*}{ POST } & Mean & SD & Mean & SD \\
\hline & 85.8938 & 33.1907 & 83.7215 & 21.0913 \\
\hline & $t=.199$ & & & \\
\hline & $P=.844$ & & & \\
\hline
\end{tabular}


- From above graphs and tables we can say that both treatments were effective for both groups.

- From above graphs and tables we can say/infer that Group A showed significant improvement as compared to Group B in Step length, Stride length, Gait velocity and Cadence.

- Gait assessment of Group A showed significant improvement at post intervention compared to Group B.

- From these Results we conclude that the Group A is better Than Group B.

\section{DISCUSSION:}

Our study aimed to improve walking. Walking is basic mobility and enhances independence to anyone. The ability to walk independently is a life enriching activity and the most efficient way of getting from one place to another in the course of our daily lives.

The temporospatial gait characteristics indicate that cadence is especially adversely affected by stroke and that the improvement in gait speed is mainly due to an increase in stride length and, to a lesser extent, to an increase in cadence.

There is significant improvement in post interventional readings of (for stride length $p=$ 0.023 , for step length $p=0.024$, for gait velocity $p=0.015$ and for cadence $p=0.024$ ) Group-A, because as per previous studies conventional therapy with motor imagery gives significant effect in intervention. S.Corina et al. says that motor imagery with conventional therapy is effective in the time difference to perform the task from pre to post-intervention. ${ }^{58}$ S.A. Zimmermann et.al says that evidence suggests Motor imagery provides additional benefits to conventional physiotherapy or occupational therapy. ${ }^{12}$ Some author says that loco motor imagery training can be considered as a useful option for restoration of ambulation for individuals with chronic hemiparetic stroke who are unable to participate in physical gait training. ${ }^{14}$ Ehrsson et al. showed an activation of specific limb-areas in the primary motor cortex. ${ }^{35}$ Motor Imagery is a dynamic state during which the representation of a specific motor action is internally activated without any motor output.

In other words motor imagery requires the conscious activation of brain regions that are also involved in movement preparation and execution, accompanied by a voluntary inhibition of the actual movement. Some author reported that the activation of the pre-supplemary motor area and the primary motor cortex during imagery of loco motor movements.

Researchers hypothesized that movement execution; motor imagery and action observation are all driven by the same basic mechanism. Motor imagery and action observation are conceived as "offline" operations of the motor areas in the brain.

Researchers also reported that better equilibrium characteristics in elderly women as measured by walking balance and foot placement measures as a result of a combined treatment of motor imagery and physical therapy.${ }^{50}$ As Motor Imagery intervention did not sufficiently modify the asymmetry that is an inherent feature of hemiparesis so it should be given with conventional therapy. ${ }^{4}$

Some researchers says that Imagery, in association with therapy, appears to be a noninvasive, efficacious complement to traditional therapy that substantially reduces impairment and improves outcomes. ${ }^{36}$

On other side there is significant improvement in Post-readings of (for stride length $p=0.000$, for step length $p=0.002$, for gait velocity $p=0.000$ and for cadence $p=0.002$ ) Group-B also because as per the previous research results, conventional therapy for stroke is effective for getting ambulation and improvement in gait. Researchers say that Task-specific activities with strength training are effective therapeutic interventions for post stroke. Possible mechanisms associated with response to therapy were related to improve motor unit activation associated with increased strength in key muscles used in gait. ${ }^{51}$ 
Authors present an intriguing hypothesis that over ground gait training such as walking forward, sideward's, may be better suited in educating patients regarding safety, while encouraging participation in therapeutic exercise to improve strength, cardiovascular fitness, movement efficiency, and agility. "Over ground gait training improves loco motor function and is a major goal of rehabilitation, and if patients want to improve walking, they need to practice walking. Over ground gait training represents the most task-specific approach in improving gait for individuals with hemiparesis after stroke. ${ }^{52}$

It was also hypothesized that Muscle strengthening and physical conditioning are to reduce impairment and disability in chronic stroke survivors. $^{53}$

Stroke rehabilitation provides a targeted and organized plan to re-learn functions lost in the shortest period of time possible. Some studies suggest that successful and meaningful recovery is more likely to be accomplished if you are dedicated and keep a high level of motivation during your rehabilitation process. ${ }^{54}$

It is recognized that repeated participation by patients in active physical therapeutic programs probably provides direct influence on the process of functional reorganization in the brain and enhances neurologic recovery. A key aspect of neural plasticity that has important implications for rehabilitation is the fact that the modifications in neuronal networks are usedependent. Clinical trials have shown that forced use and functional training contribute to improved function. ${ }^{55}$

Standardized community-based rehabilitation therapy also helps stroke patients to improve their neurological function. ${ }^{56}$

Clinical studies demonstrated that training or inpatient rehabilitation increases cortical representation with subsequent functional recovery, whereas a lack of rehabilitation or training decreases cortical representation and delayed recovery. ${ }^{57}$
Results of post readings in both groups (For pre stride length $(p=0.928)$ and post stride $(p=0.592)$, for pre step length $(p=0.777)$ and post step length $(p=0.631)$, for pre gait velocity $(p=0.459)$ and post gait velocity $(p=0.959)$, for pre cadence $(p=0.986)$ and post cadence $(p=0.844))$ show significant improvement, but improvement in Group-A was more than Group-B. In past two studies it was seen that Motor imagery provided additional benefits to conventional physiotherapy when given for upper limb functioning $^{12}$. This can be a reason for the post intervention results of the present study where the mean value of experimental group showed better results than the mean value of control group. Researchers determined that embedded $\mathrm{MI}$ (Motor Imagery with conventional therapy) is superior to added $\mathrm{MI}$ (Conventional therapy separately). Brain areas activated during $\mathrm{MI}$ and real movements show a strong congruity for single arm movements as well as complex whole body movements in stroke patients. ${ }^{6}$

These studies and researches give evidence that the Motor Imagery with conventional therapy is beneficial in gait rehabilitation. Motor imagery takes less effort and gives motivation to the subject for performing tasks hence easy to apply.

\section{CONCLUSION:}

As per the results of the present study, Motor imagery program is found to be effective when given with conventional therapy in improving gait in stroke subjects. Moreover it can be done easily by the patient as it takes less effort and motivates the subject for performing the desired task. It also does not fatigue the patient. Thus, it is a feasible method and can be applied in conjunction with conventional therapy while treating stroke patients with gait issues.

Clinical Relevance: Motor imagery program can be given together with conventional therapy to improve gait in stroke subjects and it can be done easily as it takes less effort and gives motivation to the subject for performing tasks. Thus it does not fatigue the patient. 


\section{REFERENCES:}

1. J. Donald Easton, Jeffrey L. Saver, Gregory W. Albers, Mark J. Alberts, Seemant Chaturvedi, Edward Feldmann, et.al, Definition and Evaluation of Transient Ischemic Attack, Journal of the American heart association, 2009 May;40:2276-2293.

2. Christian Weimar, Tobias Kurth, Klaus Kraywinkel, Markus Wagner, Otto Busse, Roman Ludwig Haberl, et. al, assessment of functioning and disability after ischemic stroke, Journal of the American heart association, 2002 May: 33:2053-2059.

3. O'Sullivan SB, Schmitz TJ. Physical Rehabilitation. $5^{\text {th }}$ ed. New Delhi(India): Jaypee Brothers; 2007: 705-722.

4. Ruth Dickstein, Ayelet Dunsky, Emanuel Marcovitz, Motor Imagery for Gait Rehabilitation in Post-Stroke Hemiparesis, Physical Therapy, 2004 Dec:84(24):11671177.

5. Ludmina Svetlana, M. Calayan, J Margarita, R. Dizon, A systemic review on the effective of mental practice with motor imagery in the neurologic rehabilitation of stroke patient, The internet Journal of Allied Health Sciences and Practice, 2009:7(2):1-11.

6. S. corina, B. jenny, A. Brian, K. Udo, E. Thierry, comparision of embedded and added motor imagery training in patients after stroke: study protocol of a randomised controlled pilot trial using a mixed methods approach, Trials journal, 2009:10(97): 11861745.

7. Yahagi S, Shimura K, Kasai T, An increase in cortical excitability with no change in spinal excitability during motor imagery, Hiroshima Shudo University, Japan. 1996 Aug:83(1):288-90.

8. F. Luciano, B.Giovanni, C. Laila, F. Leonardo, G. vittorio, P. Giovanni, corticospinal excitability is specifically modulated by motor imagery: a magnetic stimulation study, Science Direct,1999 Jan:37(2):147158.

9. Alfons Schnitzler, Stephan Salenius, Riitta
Salmelin, Veikko Jousmäki and Riitta Hari, involvement of primary motor cortex in maotor imagery: A neuromagnetic study, Science Direct, 1997 6(3): 201-208.

10. Guillot, Aymeric Nadrowska, Edyta Collet, Christian,using motor imagery to learn tactical movements in basketball.(report), Journal of Sport Behaviour, 2009 June.

11. H C Dijkerman, M letswaart, M Johnston, R S Mac Walter, does motor imagery training improve hand function in chronic stroke patients? A pilot study, Clinical Rehabilitation, 2004:18(5):538-549.

12. Zimmermann-Schlatter $A$, Schuster $C$, Puhan MA, Siekierka E, Steurer J, efficacy of motor imagery in post-stroke rehabilitation: a systematic review, Journal Neuroengineering Rehabilitation, 2008 Mar:14:5:8.

13. Schuster C, Puhan MA, Siekierka E, Steurer $\mathrm{J}$, efficacy of motor imagery in post-stroke rehabilitation: a systematic review, Journal Neuroengineering rehabilitation, 2008 Mar:14:5

14. Sujin Hwang, Hye- Seon Jeon, H. Y. Chung, Oh-yun Kwon, Sang-hyun Cho, Sung-hyun You, locomotor imagery training improves gait performance in people with chronic hemiparetic stroke: a controlled clinical trial, Department of Physical Therapy, College of Health Science, Yonsei University, 2010 June:24(6): 514-522.

15. Stephen M. Pribut's sports page, 2011 March.

16. JK Loudon, biomechanics of gait and running, Physiotherapy ATC,1-19.

17. Frank C. Anderson, Marcus G. Pandy, individual muscle contributions to support in normal walking, Elsevier gait posture, 2002 may:17:159-169.

18. J.S Higginson, F.E. Zajac, R.R. Neptune, S.A. Kautz, S.L. Delp, Muscle contributions to support during gait in an individual with post stroke, Journal of Biomechanics, 2006: 39: 1769-1777.

19. Deanna J. Fish, pathology forum: characteristic gait patterns in neuromuscular 
pathologies, JPO directory, 1997:9(4):163167.

20. Wolfe $C D$, Giroud $M$, Kolominsky-Rabas $P$, Dundas $R$, Lemesle $M$, Heuschmann $P$,et.al, variations in stroke incidence and survival in 3 ares of Europe, European Registries of stroke (EROS) collaboration, American stroke Association journal, 2000: 31 :2074-2079.

21. Keenan MA, Perry J, Jordan C, factors affecting balance and ambulation following stroke, Arch Phys Med Rehabilitation. 1984 Jan: 185:165-71.

22. Perry J, Garrett M, Gronley JK, Mulroy SJ, classification of walking handicap in the stroke population, American stroke association journal,1995 june: 26(6):982989.

23. Hill K, Ellis $\mathrm{P}$, Bernhardt J, Maggs $\mathrm{P}$, Hull $\mathrm{S}$, balance and mobility outcome for stroke patients: a comprehensive audit, Aust journal of physiotherapy, 1997: 43(3):173180.

24. von Schroeder HP, Coutts RD, Lyden PD, Billings $E \mathrm{Jr}$, Nickel $\mathrm{VL}$, gait parameters following stroke: a practical assessment, Journal of rehabilitation res dev, 1995 Feb: 32(1):25-31.

25. M E Morris, R lansek, T A Matyas, and J J Summers, ability to modulate walking cadence remains intact in parkinson's disease, Journal of neurology, neurosurgery \& psychiatry, 1994 Dec: 57(12): 1532-1534.

26. Corcoran PJ, Jebsen RH, Brengelmann GL, Simons BC, Effects of plastic and mental leg braces on speed and energy cost of hemiparetic ambulation, Arch Phys Med Rehabil, 1970 Feb: 51(2):69-77.

27. Gersten JW, Orr W, external work of walking in hemiparetic patients, Scand Journal Rehabilitation Medicine, 1971: 3(1): 85-8.

28. Sandra J. Olney, Carol Richardsb, hemiparetic gait following stroke, part I: Characteristics, Elsevier Gait and Posture, 1996 April: 4(2):136-148.

29. Donker SF, Beek PJ, Wagenaar RC, Mulder $\mathrm{T}$, coordination between arm and leg movements during locomotion, Journal of
Motor Behaviour, 2001 Mar: 33(1): 86-102.

30. Wagenaar RC, Beek WJ, Hemiplegic gait : a kinematic analysis using walking speed as a basis, Journal of Biomechanics, 1992 Sep: 25(9): 1007-1015.

31. Anouk Lamontagne, Sophie J. De Serres, Joyce fung, Nicole Pacuet, Stroke affects the coordination and stabilization of head, thorax and pelvis during voluntary horizontal head motions performed in walking, International Federation of Clinical Neurophysiology, 2004 July:116(1):101-111.

32. Kay CERNY, A Clinical Method of Quantitative Gait Analysis, Physical Therapy, 1983:1125-1126.

33. H.T. law, Microcomputer-based low-cost method for measurement of spatial and temporal parameters of gait, Journal of Biomedical Engineering, 1987 April:9(2):115120.

34. Winter DA, The locomotion laboratory as a clinical assessment system, Med prog technol, 1976: 4(3):95-106.

35. Sjoerd de Vries and Theo Mulder, motor imagery and stroke rehabilitation: a critical discussion, Journal Rehabilitation med, 2007: 39:5-13

36. Stephen J Page, Peter Levine, SueAnn Sisto and Mark V Johnston, A randomized efficacy and feasibility study of imagery in acute stroke, KMRREC, 2001:15:233-240.

37. Magdalena letswaart, Marie Johnston, $H$ Chris Dijkerman, Clare L Scott, Sara A Joice, Steven Hamilton, et.al, recovery of hand function through mental practice: a study protocol, BMC neurology, 2006 Oct:6:39.

38. Craig R.Hall, Wendy M. Rodgers and Kathryn A. Barr, The Use of Imagery By Atheletes in Selected Sports, Sport Psychology, 1990:4:110.

39. Yea-Ru Yang, Ray-Yau Wang, Yu-Chung Chen, Mu-Jung Kao, Dual-Task Exercise Improves Walking Ability in Chronic Stroke: A Randomized Controlled Trial, 1236-1240.

40. S. strandgaard and O.B. Paulson, pathophysiology of stroke, Journal of Cardiovascular Pharmacology, 1990: 15(1): 
838-842.

41. Joseph B. Green, Yolanda Bialy, Elena Sara, Robert W. Thatcher, An Electroencephalographic study of imagined Movement, Arch Phys Med Rehabil 1997;78:578-81.

42. Marc Jeannerod, Neural simulation of Action: Aunifying Mechanism for Motor Cognition, Science Direct, 2001 july :14(1): 103-109.

43. Wehner T, Vogt S, Stadler M, task specific EMG- Characteristics during mental training, Psycho Res, 1984:46(4):389-401.

44. M. Lotze, G. Scheler, H.-R.M. Tan, C. Braun and N. Birbaumer, The musician's brain: functional imaging of amateurs and professionals during performance and imagery, Science Direct, 2003: 1817-1829.

45. Rushall, B. S., \& Lippman, L. G, introduction to imagery in physical performance, International Journal for Sport Psychology, 1997:29:57-72.

46. Philip L. Jackson, Julien Doyon, Carol L. Richards, Francine Malouin, The Efficacy of Combined Physical and Mental Practice in the Learning of a Foot-Sequence Task after Stroke: A Case Report, Neurorehabil Neural Repair, 2004 :18(2):106-111.

47. Melanie Gregg, Craig Hall and Andrew Butler, movement imagery questionnaire revised second (miq-rs), Advance Access Publication, 2010: 7(2):249-257.

48. Susan L Morris, Karen J Dodd, Meg E Morris, outcomes of progressive resistance strength training following stroke: a systematic review, Clinical Rehabilitation, 2004 Jan:18 (1) 27-39.

49. Catherine M. Dean, Carol L. Richards, Francine Malouin, Task-Related Circuit Training Improves Performance of Locomotor Tasks in Chronic Stroke: A
Randomized, Controlled Pilot Trial, The American Academy of Physical Medicine and Rehabilitation, 2000:81:409-417.

50. Th. Mulder, Motor imagery and action observation: cognitive tools for rehabilitation, J Neural Transm, 2007:114: 1265-1278.

51. Sullivan K, Klassen T, Mulroy S, Combined task-specific training and strengthening effects on locomotor recovery post-stroke: a case study, Journal of Physical Therapy, 2006 sep: 30(3): 130-141.

52. Lewek, Michael D. he Value of Overground Gait Training for Improving Locomotion in Individuals with Chronic Stroke, Journal of Neurologic Physical Therapy, 2009 Dec:33(4):187-188.

53. Teixeira-Salmela LF, Olney SJ, Nadeau S, Brouwer $B$, Muscle strengthening and physical conditions to reduce impairment and disability in chronic stroke survivors. Arch Phys Med Rehabi,. 1999 Oct;80(10):1211-1218.

54. Jose Vega, Stroke Rehabilitation is a Critical Part Stroke Recovery, 2009 Sept.

55. Auri Bruno-Petrina, motor recovery in Stroke, Physical therapy and rehabilitation, 2010 Sept.

56. Jian Jun Yu, Youg Shan yu, Yi hu, Wen Hua Chen, YuLian zhu, Xiao Cui,et.al, the effects of community- based rehabilitation on stroke patients in china: a single-blind randomised controlled multicentre trial, clinical rehabilitation, 2009 April:23(5), 408417.

57. Joel Stein, Richard L. Harvey, Richard F. Macko, Carolee J. Winstein, Richard D. Zorowitz, stroke recovery and rehabilitation, google book, 2009: 591 